\title{
Absolute Values of Surface and Step Free Energies from Equilibrium Crystal Shapes
}

\author{
H. P. Bonzel* and A. Emundts \\ Institut für Grenzflächenforschung und Vakuumphysik, Forschungszentrum Jülich, D-52425 Jülich, Germany
}

(Received 9 December 1999)

\begin{abstract}
It is shown that exact images of the three-dimensional equilibrium shape of crystallites (ECS), recorded at several temperatures between 0.3 and 0.8 of the melting temperature of a solid, can be evaluated to yield absolute values of the surface and step free energies versus temperature, in addition to the formation energy of kinks. The essential input for this novel approach is the temperature variation of the size of a facet on the ECS and of the separation between the Wulff point and that particular facet. This approach promises access to surface free energies over a large temperature range and for well-defined low-index surface orientations.
\end{abstract}

PACS numbers: 68.35.Md, 68.35.Bs, 82.65.Dp

The absolute value of the surface free energy of solid materials is a fundamentally important energetic quantity which is needed for the understanding of a large number of basic and applied phenomena, such as crystal growth, surface faceting, growth and stability of thin films, the shape of small crystallites in a supported catalyst, and many general materials science applications. Despite its wellrecognized significance, there are relatively few reliable primary data of experimental surface free energies because they are very difficult to measure. Only a few techniques, such as the zero-creep [1-4] and the cleavage techniques [5], have been used repeatedly in the past to obtain quantitative values for a limited number of solids, mostly metals. "Recommended" values of surface energies of metals have been derived in a phenomenological systematic comparison of some experimental solid surface free energies and liquid metal surface energies for a large number of elements [6]. All of the known values are considered to be averaged over a range of crystallographic orientations. Finally, very little is known about the temperature dependence of the surface free energy of solids since most experimental data were obtained at temperatures near the melting point.

In this Letter we propose to utilize the equilibrium crystal shape (ECS) of a solid crystallite for the quantitative determination of absolute surface energies as well as step free energies for a range of temperatures well below the melting temperature. The starting point is an exact image of portions of the ECS at a given temperature, obtained, for example, by high resolution scanning tunneling microscopy (STM) $[7,8]$. In previous work we have demonstrated how, at a single temperature, the relative formation energies of kinks and steps as well as the step interaction energies of vicinal (111) surfaces can be determined from the regular ECS of small $\mathrm{Pb}$ crystallites [9]. We propose now, that analyzing such a crystallite at several temperatures by STM and measuring the facet and crystal size versus temperature, the absolute values of kink formation, step formation, and surface free energy of the low-index facet orientation can be evaluated, as long as the ECS is continuously differentiable, i.e., exhibiting all crystallographic orientations.
The ECS of a solid is governed by the temperature dependent anisotropic surface free energy which in turn depends on step and step interaction energies [10-13]. Figure 1 illustrates schematically the geometric relationship between the orientation dependent surface free energy and the ECS in a two-dimensional Wulff plot. Surface and step free energies are related to each other by length ratios in the ECS, such as the separation between the center of the crystallite (Wulff point 0 ) and the facet, $z_{0}$, and the distance between the center of the facet and the edge of the facet, $|r(T)|$, in a direction perpendicular to low-index steps of surfaces vicinal to the facet $[11,12]$. With increasing temperature, the diameter of a facet becomes smaller, corresponding to a decreasing step free energy. The main reason for the decreasing step free energy is the excitation of kinks, possible at any finite temperature.

Lattice model calculations in the framework of an Ising model and various forms of the solid-on-solid model have illustrated the thermal evolution of two- and threedimensional ECSs in great detail, providing the theoretical background for the orientation and temperature dependent surface and step free energies and also for the relationship between step free energy and facet radius [11,14-17]. Lattice theory in combination with extensive Monte Carlo simulations has also produced a realistic view of the temperature dependent ECS [14,18,19]. For regular shapes

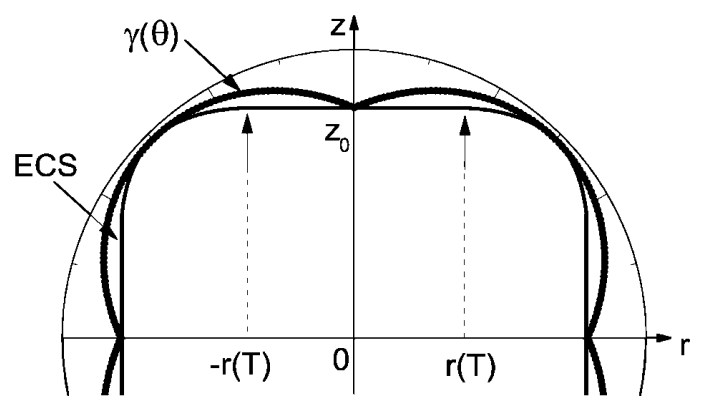

FIG. 1. Schematic of the equilibrium crystal shape (ECS) cross section, together with the anisotropic surface free energy, $\gamma(\theta)$, drawn in a polar plot (Wulff plot). A facet is outlined by $r(T)$. The separation between the facet and the center of the plot is $z_{0}$. 
it is understood that the facet radius in a high symmetry direction is a direct measure of the free energy of an isolated step. Considering such a single step in a 2D Ising or solid-on-solid model, the temperature dependence of its free energy, $f_{1}(T)$, regulated by the kink formation energy, $\varepsilon_{k}$, is given by the following expression $[13,15,16,20]$ :

$$
f_{1}(T)=f_{1}(0)-k T \ln \left[\operatorname{coth} \frac{\varepsilon_{k}}{2 k T}\right],
$$

where $f_{1}(0)$ is the step energy at $0 \mathrm{~K}$. Compared to results obtained by $3 \mathrm{D}$ lattice calculations, this equation is estimated to be valid at least for temperatures below about $70 \%$ of the roughening temperature of the nearest facet, $T_{R}=1.135 \varepsilon_{k} / k[14,15,18]$. The kink formation energy is here assumed to be temperature independent.

Vibrational motion of step atoms, in particular, normal to the surface, is also likely to be important [21-24], especially at lower temperatures where kink excitation is still negligible. To account for this effect, we approximate the vibrational free energy of step atoms by an Einstein model where the dominant contribution of the spectral density of surface atom vibrations perpendicular to the surface is considered. The vibrational free energy of step atoms is understood to be an excess energy [25], relative to that of a flat terrace. We include here for the first time this additional free energy in Eq. (1), rewriting it in terms of relative energies and approximating the $\ln \operatorname{coth}(x)$ function by $2 \exp (-2 x)$, valid for $\exp (-4 x) \ll 1$. This yields

$$
\begin{aligned}
\frac{f_{1}(T)}{f_{1}(0)}=1-\frac{2 k T}{f_{1}(0)}\left\{\exp \left(-\frac{\varepsilon_{k}}{k T}\right)\right. \\
\left.-\frac{3}{2} \ln \frac{1-\exp \left(-\frac{\Theta_{s}}{T}\right)}{1-\exp \left(-\frac{\Theta_{t}}{T}\right)}\right\},
\end{aligned}
$$

where $\Theta_{s}$ and $\Theta_{t}$ are the Einstein temperatures of step and terrace atoms, respectively. For $T \gg \Theta$ the vibrational free energy is $3 k T \ln \left(\nu_{s} / \nu_{t}\right)$, with $\nu_{s}$ and $\nu_{t}$ being characteristic frequencies of step and terrace atoms, respectively. We assume the ratio of these frequencies to be equal to $\sqrt{N_{s} / N_{t}}$ where $N_{s}$ and $N_{t}$ are the respective coordination numbers. For close-packed steps on a fcc(111) vicinal surface, the logarithmic term in Eq. (2) amounts then to 0.188 . To visualize the effect of surface vibrations on the temperature dependence of the step free energy, we show a comparison of Eqs. (1) and (2) in Fig. 2 for a set of reasonable parameters. For this purpose we introduce a relative temperature scale, $T / T_{M}$, where $T_{M}$ is the absolute temperature of melting. We have examined recent experimental and theoretical data of step energies [26], mostly for the $\langle 110\rangle$ oriented steps on (111) metal surfaces, e.g., for $\mathrm{Al}$ [27], $\mathrm{Pb}$ [9], $\mathrm{Cu}[28,29]$, and $\mathrm{Pt}$ [30], and have chosen an average value of $f_{1}(0) / k T_{M}=2.5$. For the kink energy we assume a value of $0.4 f_{1}(0) / k T_{M}$, i.e., $\varepsilon_{k} / k T_{M}=1.0$. The calculated functions in Fig. 2 illustrate that the onset of vibrational entropy occurs

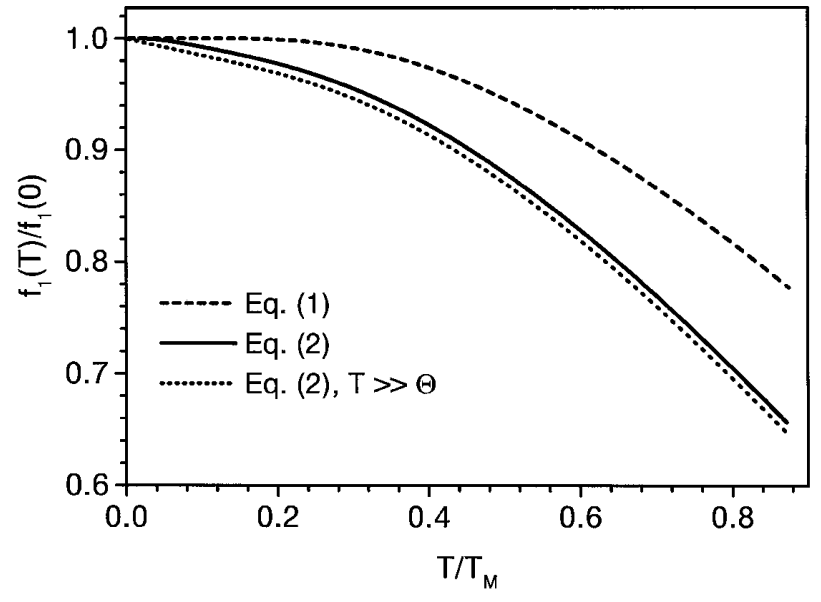

FIG. 2. Plot of the relative step free energy, $f_{1}(T) / f_{1}(0)$, versus relative temperature, $T / T_{M}$, according to Eqs. (1) and (2). Relative Einstein temperatures, $\Theta / T_{M}$, of 0.120 for step atoms and 0.136 for terrace atoms have been chosen (solid line). In the high temperature limit of the Einstein model, the ratio of Einstein frequencies was set equal to $\sqrt{7 / 9}=0.882$ (dotted line). A value of $f_{1}(0) / k T_{M}=2.5$ and a relative kink energy of $\varepsilon_{k} / k T_{M}=1.0$ have been selected in all cases.

at much lower temperatures than that of the configurational entropy due to kink formation. Vibrational effects are thus likely to be important for the entire range of temperature. On the other hand, the high temperature limit of the Einstein vibrational free energy is a good approximation which we adopt from now on.

To relate the expression in Eq. (2) to experiment, we replace $f_{1}(T) / f_{1}(0)$ by $r(T) / r(0)$, recognizing that a ratio of step energies is equal to a ratio of corresponding facet radii $[11,12]$, taken for the same facet at the same azimuth. We now describe how Eq. (2) in the high temperature limit can be applied towards the evaluation of absolute step and kink formation energies of a single step. Note that the step free energy as related to the facet radius is by definition that of a solitary step, consistent with using Eq. (2).

Experimentally, the variation of the radius $r(T)$ of a low-index facet with temperature can be readily measured by a suitable STM, capable of imaging the same crystal over a range of temperatures, e.g., between 0.3 and 0.8 of the absolute melting temperature, $T_{M}$. The kinetics of equilibration are expected to be sufficiently fast for crystallites of the size 200-1000 nm diameter, for the smallest ones even at $0.3 T_{M}$ or below. Let us assume that a number of facet radii versus temperature on an equilibrated crystal have been determined. With the relative energy scale introduced for kink and step formation, such as $\varepsilon_{k} / k T_{M}$ and $f_{1}(T) / k T_{M}$, we transform Eq. (2) into the form

$$
\begin{aligned}
\left(1-\frac{r(T)}{r(0)}\right) \frac{T_{M}}{T}=\frac{2 k T_{M}}{f_{1}(0)} & \left\{\exp \left(-\frac{\varepsilon_{k}}{k T_{M}} \frac{T_{M}}{T}\right)\right. \\
& \left.-\frac{3}{2} \ln \sqrt{\frac{7}{9}}\right\} .
\end{aligned}
$$


There are a total of three unknowns in Eq. (3): $r(0), f_{1}(0)$, and $\varepsilon_{k}$. All of these can be obtained by an iterative process. At first, we assume $r(0)$ as the value measured at the minimum temperature, $r\left(T_{\min }\right)$. Secondly, according to a plot of $\ln \left\{\left(T_{M} / T\right)[1-r(T) / r(0)]\right\}$ versus $T_{M} / T$, Eq. (3), an approximate value of $f_{1}(0) / k T_{M}$ is obtained from an extrapolation to $T_{M} / T=0$. This value is used to calculate the vibrational part of the free energy, $\alpha_{v}=0.188\left[2 k T_{M} / f_{1}(0)\right]$, which is added to the left side of Eq. (3). If we now plot $\ln \left\{\left(T_{M} / T\right)[1-r(T) / r(0)]+\right.$ $\alpha_{v}$ \} versus $T_{M} / T$, a nearly straight line should result, with the ordinate intercept being equal to $\ln \left[2 k T_{M} / f_{1}(0)\right]$, and the slope of the line equal to $\varepsilon_{k} / k T_{M}$. With the new value of $f_{1}(0) / k T_{M}$, a more accurate value of $r(0)$ can be accessed through $r(0)=r\left(T_{\min }\right) f_{1}(0) / f_{1}\left(T_{\min }\right)$, and step number two can be repeated. A few iterations of this kind should yield the absolute step energy at $0 \mathrm{~K}$ and the kink formation energy $\varepsilon_{k}$ for that step. Of course, having $f_{1}(0)$ and the kink energy, the variation of the step free energy $f_{1}(T)$ with temperature, given by Eq. (2), is also known over a large range.

Once the step free energy has been determined for a certain temperature, the surface free energy for the facet orientation, $f_{0}(T)$, at the same temperature, is easily obtained from the three-dimensional ECS. For regular shapes we use the important relationship mentioned above [11,12], namely, that

$$
\frac{f_{0}(T)}{z_{0}(T)}=\frac{f_{1}(T)}{r(T)} .
$$

This means that the surface free energy $f_{0}(T)$ can be calculated from the length ratio $z_{0}(T) / r(T)$ and the step free energy $f_{1}(T)$ at $T$. The length $z_{0}$ can be evaluated from the ECS image by locating the center of the crystallite and measuring its distance to the facet. Note that the temperature variation of $z_{0}$ with $T$ is to a first order negligible, because the crystal volume stays constant at all temperatures and the maximum anisotropy of the surface free energy is small. A better approximation is to treat the missing volume due to facets as sphere segments and calculate a function $r\left(z_{0}\right)$ by assuming a number $n$ of equal segments, representing $n$ facets, on the ECS. The result is

$$
r\left(z_{0}, T\right)=\left[\frac{4 r_{s p h}^{3}}{3 n z_{0}}-\frac{4 z_{0}^{2}}{3 n}\right]^{1 / 2} .
$$

Figure 3 shows a plot of $z_{0}(r)$ versus $r(T)$ for $n=6$ and 8 , respectively. Note that $z_{0}$ decreases by only $16 \%$ for a 16-fold increase in facet radius $r$, for a fictitious change in temperature from near $T_{M}$ to $0 \mathrm{~K}$. A ratio of $r / z_{0}=f_{1}(0) / f_{0}(0)=0.34-0.4[26,31]$ marks the low temperature end. The dependence in Fig. 3 can be used to make a correction for the temperature dependence of $z_{0}(T)$, at least for those temperatures where $z_{0}(T)$ cannot be obtained experimentally because of kinetic limitations. The approach described in this Letter yields then for the

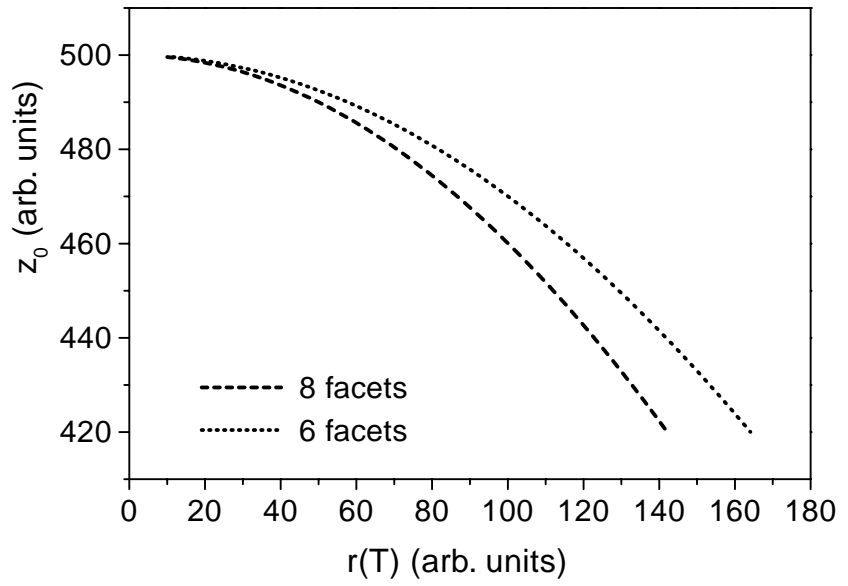

FIG. 3. Plot of the separation between facet plane and Wulff point, $z_{0}(T)$, versus the facet radius, $r(T)$. The calculation assumes six and eight circular facets, respectively, each of which cuts away a spherical segment from a perfect sphere (equivalent to the ECS in the liquid state). The radius of the sphere is 500 units. The volume of the truncated sphere (ECS) is kept constant and equal to that of the complete sphere in the liquid state. High values of $r(T)$ correspond to low temperature.

first time reliable absolute values of the surface free energy over a considerable range of temperatures, even down to low temperatures near $0.3 T_{M}$. If the correction to $z_{0}(T)$ is made, according to Eq. (5), the absolute surface energy of a facet orientation can in principle be obtained even at $0 \mathrm{~K}$. This is another important feature because no other experimental approach yields surface energies at $0 \mathrm{~K}$. On the other hand, such values are needed for a direct comparison with corresponding a priori theoretical values.

The advantages of deriving absolute step and surface free energies from accurate three-dimensional images of the ECS are manifold. First, utilizing a modern variable temperature STM, possibly in combination with scanning electron microscopy [32], makes investigations of small crystallites at temperature feasible. Facets and their changing size with temperature can be readily measured. Second, comparing the experimental effort of a clean zero-creep experiment [3] to STM imaging of small crystallites, the latter carried out under ultrahigh vacuum conditions and excellent surface cleanliness control, makes the currently proposed new technique to be the first choice. A similar argument holds for the comparison with cleavage experiments which are, in any case, feasible for only a small number of materials. Third, the approach outlined in this Letter yields specific energies of well-defined crystallographic orientations, something that is very difficult to achieve in a zero-creep study of thin foils. Fourth, the technique of evaluating surface and step free energies from ECS allows a much larger range of variables, such as temperature, orientation, adsorbates, thin overlayers, etc. In particular, the study of the effect of adsorbed species on surface and step free energies is a largely neglected area of research, but most interesting 
in terms of Gibbs' thermodynamic theory, and should become feasible with the newly proposed technique. A final advantage should also be noted, namely, that the anisotropy of the step and surface free energies is also given by the ECS $[9,33]$.

Finally, we may add that a variation of the technique can also be applied to small mounds created on a flat crystal substrate (e.g., by vapor deposition or lithography). Although these mounds are nonequilibrium features, it is conceivable that they may exhibit quasiequilibrium shapes near their tops, in particular, if those are faceted. Then the same procedure outlined above for the three-dimensional ECS can be applied to the top facet of the mounds, leading to the determination of kink and absolute step free energies. Absolute surface free energies, however, cannot be obtained in this way. This approach would complement a recent proposal for evaluating the absolute step free energy from the temperature variation of equilibrated twodimensional adatom or vacancy islands $[28,34]$.

We are grateful to Walter Selke for a discussion of lattice theory in context of Eq. (1). We also thank Harald Ibach for numerous valuable discussions and his critical reading of the manuscript.

*Corresponding author.

Email address: h.bonzel@fz-juelich.de

[1] E. D. Hondros, Proc. R. Soc. London A 286, 479 (1965).

[2] E. D. Hondros and M. McLean, in Proceedings of the Conference on Structure et Propriétés des Surfaces des Solides (CNRS, Paris, 1969).

[3] E. D. Hondros, in Physicochemical Measurements in Metals Research, edited by R. A. Rapp, Techniques of Metals Research Vol. IV (Interscience, New York, 1970), Pt. 2, pp. 293-348.

[4] V. K. Kumikov and K. B. Khokonov, J. Appl. Phys. 54, 1346 (1983).

[5] J.C. Bilello, D. Dew-Hughes, and A. T. Pucino, J. Appl. Phys. 54, 1821 (1983).

[6] A. R. Miedema, Z. Metallkd. 69, 455 (1978).

[7] S. Surnev, K. Arenhold, P. Coenen, B. Voigtländer, H. P. Bonzel, and P. Wynblatt, J. Vac. Sci. Technol. A 16, 1059 (1998).

[8] K. Arenhold, S. Surnev, P. Coenen, H. P. Bonzel, and P. Wynblatt, Surf. Sci. 417, L1160 (1998).
[9] K. Arenhold, S. Surnev, H. P. Bonzel, and P. Wynblatt, Surf. Sci. 424, 271 (1999).

[10] E. F. Gruber and W. W. Mullins, J. Phys. Chem. Solids 28, 875 (1967).

[11] C. Jayaprakash and W. F. Saam, Phys. Rev. B 30, 3916 (1984).

[12] M. Wortis, in Chemistry and Physics of Solid Surfaces, edited by R. Vanselow and R. Howe (Springer-Verlag, New York, 1988), Vol. 7, pp. 367-405.

[13] E. D. Williams, R. J. Phaneuf, J. Wei, N.C. Bartelt, and T. L. Einstein, Surf. Sci. 294, 219 (1993).

[14] H. van Beijeren, Phys. Rev. Lett. 38, 993 (1977).

[15] C. Rottman and M. Wortis, Phys. Rev. B 24, 6274 (1981).

[16] J.E. Avron, H. van Beijeren, L. S. Schulman, and R. K. P. Zia, J. Phys. A 15, L81 (1982).

[17] C. Rottman and M. Wortis, Phys. Rev. B 29, 328 (1984).

[18] K. K. Mon, S. Wansleben, D. P. Landau, and K. Binder, Phys. Rev. Lett. 60, 708 (1988).

[19] A. Trayanov, A. C. Levi, and E. Tosatti, Surf. Sci. 233, 184 (1990).

[20] H. J. Leamy, G. H. Gilmer, and K. A. Jackson, in Surface Physics of Materials, edited by J. M. Blakely (Academic Press, New York, 1975), Vol. 1, pp. 144-148.

[21] L. Dobrzynski and J. Friedel, Surf. Sci. 12, 469 (1968).

[22] G. Tréglia and M.-C. Desjonquères, J. Phys. (Paris) 46, 987 (1985).

[23] A. Kara, S. Durukanoglu, and T. S. Rahman, Phys. Rev. B 53, 15489 (1996).

[24] S. Durukanoglu, A. Kara, and T. S. Rahman, Phys. Rev. B 55, 13894 (1997).

[25] M.-C. Desjonquères and D. Spanjaard, Concepts in Surface Physics (Springer-Verlag, Berlin, 1996), p. 136 ff.

[26] L. Vitos, H. L. Skriver, and J. Kollár, Surf. Sci. 425, 212 (1999).

[27] R. Stumpf and M. Scheffler, Phys. Rev. Lett. 72, 254 (1994).

[28] G. Schulze Icking-Konert, M. Giesen, and H. Ibach, Phys. Rev. Lett. 83, 3880 (1999).

[29] P. Feibelman, Phys. Rev. B 60, 11118 (1999).

[30] G. Boisvert, L. J. Lewis, and M. Scheffler, Phys. Rev. B 57, 1881 (1998).

[31] H. P. Bonzel, Surf. Sci. 328, L571 (1995).

[32] A. Emundts, P. Coenen, B. Voigtländer, G. Pirug, and H. P. Bonzel (to be published).

[33] J. C. Heyraud and J. J. Métois, Surf. Sci. 128, 334 (1983).

[34] D.C. Schloesser, L. K. Verheij, G. Rosenfeld, and G. Comsa, Phys. Rev. Lett. 82, 3843 (1999). 